\title{
Making Methods Sociable: Dialogue, Ethics and Authorship in Qualitative Research
}

\begin{abstract}
The article argues for fostering sociable forms of dialogue in qualitative research. Conventional research shares an emphasis on extracting narratives with judicial and invasive state modes of enquiry rather than on learning from a genuine two-way dialogue between participants and researchers. Using a study of young migrants we show how involving participants as observers and shapers of analytical dialogue can produce circulations of communication oscillating across the researcher's and participant's horizons of understanding. This produces new insight, beyond the limits of qualitative investigation, that extracts information from participants, and in so doing has the potential to affect shifts in perception that animate and enchant experience. It has consequences for re-thinking authorship that share, credit and specify responsibility. Developing such an approach opposes the 'ethical hypochondria' characterising qualitative research culture, where 'automatic anonymity' is limiting the potential of research to travel, connect people and engage the public imagination.
\end{abstract}

\section{KEYWORDS}


Authorship/auto-ethnography/ethics/migration/participatory methods /

\section{WORD COUNT $(7,695)$}

\section{Introduction: How Do you Know That?}

Rule one in the practical 'how to' guide to doing interview research is first to find a quiet place. Preferably the information gathering should also be private and not overheard. This is not just an ethical matter concerning confidentiality but a practical one. Noise makes the clean transcription of a human voice captured on the digital recorder more difficult. The problem with thinking of social research in this way - as the mere transposition of talk to text data - is that it is not very sociable. The conditions of research that make such a clinical act possible are also antithetical to the establishment of a genuine two-way dialogue. For some participants, like the young migrants discussed in this article, these kinds of interviews are often more in keeping with being questioned by the police or immigration officers. This can be a particular concern when working with marginalised and vulnerable groups.

The impulse to transpose talk to text data in a 'clean' fashion has a history. Ray Lee pointed out that in the early days of social research, before the invention of tape-recorders, participants would have to come 
into the university to have their words transcribed in real-time. The renowned Chicago School sociologist Clifford Shaw collected hundreds of life histories from young people using a stenographer hidden behind a screen, in a scene reminiscent of a court-room (Lee 2004: 72).

We did not want our study of the experience of young adult migrants to be conducted under conditions redolent of the criminal justice system or the immigration service. This is because we did not want to reflect the forms of coercive scrutiny that participants had already experienced from the state. Instead we wanted to widen the parameters of exchange beyond those predicated on surveillance and suspicion, to encourage exchange and dialogue. As such we offered young people the opportunity to become observers of their own lives through writing, taking photographs and keeping journals and scrapbooks. When we met participants to talk about what they made, we often did so in public in cafés or restaurants (even though the sociable background noise of the cafe drove the patient secretaries of our transcription service to distraction).

A favourite meeting place was The Eatery in Leytonstone, east London. In early October 2011 we arranged to meet Charlynne Bryan, one of our most dedicated participants, there to talk about a draft journal article we had written based on her life. She had assembled a scrapbook about her experiences and life in London. This included cut-outs from magazine articles, photographs she had taken, pictures she had drawn and her 
poems and creative writing. We talked about this scrapbook and a range of issues connected to it over eight meetings with her during the three-year period of the study. She used the scrapbook to chronicle her life as well as to direct our attention towards the themes she was exploring. As the project was drawing to a close we arranged to meet in The Eatery to talk about how we used one of Charlynne's poems to illustrate the new hierarchies and divisions amongst migrants emerging in London.

In our discussions at The Eatery we shuttled between our very different cultural and historical experiences. In the crude labels of identity categories it was a meeting with Author A, a British Indian sociologist and Author B, a white male English sociologist (authors' names removed throughout for blind peer review process), and Charlynne who came to Britain from Dominica as a student, aged 18. Charlynne read the article and made notes on it for discussion. She attended the University of East London obtaining a first class degree in Psychosocial Studies in 2009. Reading an academic paper was a familiar exercise for her. We sat down, ordered some samosas and started talking about the relationship between what she had made for the project and our argument.

After about twenty minutes of thought-provoking conversation Author A interrupted proceedings by going to the toilet. We tried to pause working through Charlynne's points so that he would not miss anything important. Charlynne turned to Author B and said: "What amazed me and 
I kept thinking reading the paper was the sense of - 'How do they know that?" She seemed genuinely surprised we had come to this accurate understanding, as if reading the paper somehow resulted in a kind of enchantment with familiar things that she had taken for granted. Author B answered simply, "Because you told us".

This fieldwork fable encompasses our argument for sociable methods that are participatory and dialogic. In the dialogues taking place over three years, Charlynne helped us notice things we would otherwise not have seen without her insight and her analytical choices concerning both what to observe and the means by which she was going to conduct her observations. Equally, assembling her perspectives with theoretical ideas that the researchers helped to raise led her to think again about her relationship to the past, present and future. Consequentially, she reevaluated those relationships, and her past, from the vantage point of the here and now. In these dialogues, we re-imagined empirical enquiry in a way that blurred the relationship between observer and observed, data and analysis and participants and authors.

These ways of working with rather than on participants extended our sociological horizon. For other participants their involvement in the project altered their relationship to themselves and both their and our understanding of their social position. In what follows we reflect on this experience through developing a broader argument about the politics of 
method, the craft of research and the possibility of new kinds of authorship and forms of sociological circulation. Before exploring these issues we first want to examine the politics of method with regard to researching migration.

\section{Figuring the Migrant}

In the introduction to Black Skins, White Masks Franz Fanon (1967:14) comments that it is "good form" at the commencement of any work of social investigation to provide a "statement of its methodological point of view". He warns his readers that if they anticipate this then they are going to be disappointed. "I shall be derelict" he writes. "I leave methods to the botanists and the mathematicians. There is a point at which methods devour themselves". Fanon's sceptical irreverence regarding research methodology is, in part, due to the fact that, for him, its cold procedural logic foreclosed passionate political critique. This is reminiscent of $\mathrm{C}$ Wright Mills' (1959:246) warning against the limits of what he called the "fetishism of method and technique". We would like to make two quite different points that follow from these observations. First, Fanon's allergic reaction to social science methods concerns how they produced knowledge used to maintain both colonial power relations and myths of racial difference supporting this. George Steinmetz (2009) has shown that sociology was deeply entangled in the colonial encounter, no less profoundly than social anthropology, despite the reluctance to face this 
legacy in the discipline. Additionally, within the sociology of race and ethnic relations an intense debate took place during the 1980s about the role of social science in producing pathological accounts of minority communities, reducing them to either 'victims' or 'problems' (Gilroy 1987). Current times dictate that it is worth re-examining the ways in which the frameworks for understanding migration and in which the figure of the migrant is produced in debate have become part of the problem itself.

In a study of the representation of asylum seekers Imogen Tyler (2006:191) argued that the process of social abjection works precisely through the repeated "constitution of the figure of the asylum-seeker as a threat". The stigmatised are constructed not only as qualitative caricatures but also through the process of literal figuring and enumeration. Journalists and politicians protest that there are simply too many migrants. On Thursday November $18^{\text {th }}, 2011$ the Daily Express newspaper ran the headline " $4 \mathrm{~m}$ Migrants Work in UK: No wonder jobs are drying up." Tyler adds that Left or liberal commentators also invoke the figure of the asylum seeker in restrictive ways. She shows that, from humanitarian advocates to cultural theorists and philosophers, the condition of the refugee is used as an abstract analogy. This has important limiting consequences.

Author Tom L. Friedman, Foreign Affairs columnist for the New York Times, exemplifies this, albeit in reference to immigrants more broadly 
rather than asylum seekers. Over a period of time Friedman has advocated de-regulating immigration in order to stimulate economic growth. In September 2011 he gave a presentation to the IBM Think Forum in which he proposed that the attributes associated with the figure of the immigrant could represent a universal economic fix. He said:

We are all new immigrants, Friends, we are all new immigrants to the hyper connected world. And how does the new immigrant think? He or she starts everyday and says "nothing is owed me, nothing is owed me in this world, there is no legacy place waiting for me at IBM or Harvard or my state university. I'd better figure out what world I am in and understand where the opportunities are and work absolutely harder than the next guy. I am a new immigrant and nothing is owed me here'. We are all new Immigrants.

There are many things that are interesting in Friedman's combination of neo-liberal economics and pro-immigrant rhetoric. What we want to foreground here is that the universalising claim that 'we are all immigrants' occludes and erases the actual experience of migrants. The confinements and restrictions that are also part of the hyper-connected world described by Friedman disappear from view. As Tyler (2009:199-200) comments: "If analogy, generalisation and abstraction take the place of listening and translation [...] then what is forgotten are the bodies and 
constituencies from whose suffering such accounts takes its cues".

Part of our impulse towards thinking about how we listen comes from realising that many of our participants were likely to have been subject to coercive forms of qualitative interview, which we needed to avoid replicating. Modes of interview informed by social science but practiced by immigration authorities and the police can be focused on transforming personal history into information about times, places, and circumstances, the truth of which can be verified or not. As a high ranking civil servant told Author B during the South London Citizens' investigation into the immigration service: "Well, what we want is to find the best way to extract the asylum narrative". The use of the notion of 'narrative' here shows how methodological language has become implicated in state and judicial modes of enquiry into social life. The Civil Servant's comment is reminiscent of Kvale and Brinkmann's (2009) metaphor of the interviewer as a miner, digging into the subject's secret interior world.

The process of mining and extracting is invasive. Something is taken out of you. This is reflected in the way in which, during the investigation, migrants at the UK Border Agency in Croydon often spoke about experiences of rape or violence as they sat in an austere environment similar to a bank teller's kiosk. Within the regulatory regimes of the immigration service narrative methods are being supplemented by biometric techniques. The fingerprint is the most common example but 
with the digitization of physiological and physical attributes biometric data has been expanded to include retinal scans, facial thermography, gait analysis and even DNA and brainwave biometrics. As Benjamin J. Muller (forthcoming) has argued "A central aim of biometric technology is verifying that: you are who you say you are".

Participatory techniques offer the potential to shift the parameters of knowledge production for people who are often scrutinized. This is closer to Kvale and Brinkmann's (2009:48) second metaphor of the interviewer as a traveller, recalling the original Latin meaning of conversation as a 'wandering together with'. However, before discussing our own attempts to do this we want to point out some caveats about participatory methods.

There is a danger in qualitative research of assuming that there is a 'technological fix' for capturing the lived textures of social life through the use of digital and audio-visual techniques (see for example Pauwels 2012). 'Multi-media' techniques like photo-elicitation can result in a kind of pseudo-participatory form of research that complies with the dominant practice of interviewing in social research where the interviewer remains in control of the topics, order and structure of the research dialogue (Gibson and Brown 2009). Our attempt at constructing a sociable research dialogue was informed by elicitation techniques, which broadened the parameters of qualitative inquiry by offering participants a choice/set of choices of what to record/observe for research, often drawing on a range of 
audio-visual techniques (Mason and Davies 2009). As such it becomes recognisable to an extent that knowledge is a co-construction of researchers and participants, at times leading to the adoption of a participatory model of research, inviting participants to comment on design, methods and dissemination (Bagnoli and Clark 2010).

It has been argued that these techniques have given a voice to research participants which breaches the manner in which information is often extracted from them, illuminating 'backstage' areas of participants' lives which would otherwise have not been explored because researchers would not have known or thought to prompt about them (Muir and Mason 2012). However, elicitation techniques involve researchers defining the task/s and method for participants, whether through the photography of places one might like to go or not, favourite pastimes, memorable sights from a daily walk to or from home and so on (Wright et al 2010). The role of the researcher/s in defining the task and method and in analysis of the fieldwork is premised on decisions they make about what to explore and review to meet research objectives, even if participants have autonomy within this.

Elicitation and interview methods as described above exist within a particular epistemological paradigm. This is where researchers have an expertise in relating participant's observations/accounts to research objectives and public affairs, with participants positioned as 
knowledgeable about their own lives and what their world means to them. They narrate this according to the criteria or questions provided by interviewers. Put simply researchers ask questions and participants respond.

We argue for a form of research that shifts the ordering of researcher dialogue, where participants are involved in deciding what/how methods are to be used to address research objectives. Distinctively, this includes involving participants in making analytical choices over what topics are important and what data needs to be collected. In our research we attempted to re-configure this paradigm by blurring the posited division of labour between researchers and participants/interviewees, premising both extraction and elicitation methods. This was by deciding individually with research participants how and what to explore analytically in meeting research objectives and also in an analysis of the material created from the study by participants and by us.

Some participants chose not to take the opportunity to suggest what methods might be used to address research objectives or to have an analytical conversation over what topics were important to collect material on. Instead, some appeared to simply want to respond to our questioning in a conventional way, although even here some of those would show us photographs, mementos, places in their neighbourhood and so forth that they had suggested we see, given the topics our encounters were exploring. 
As a result some participants chose to be 'mined for research data'. However, we offered the chance to expand the parameters of their possible involvement distinguishing our approach from both data extraction and pseudo-participatory techniques. This in itself was a movement toward sociable dialogue in research.

In the form of research we propose, dialogue and understanding are produced through what Gadamer (2004: 305) called a 'fusion of horizons'. For us this occurs where researchers and participants come together to compare what each has added and missed at each stage, given that neither researchers nor participants have transparent access to the truths of public or private issues. This results in the forging of a relationship whereby researchers can make observations about participants' personal worlds and participants can shed light on how issues in their personal worlds connect with public issues.

We explained our study to potential participants as being about the lives young migrants were leading and wanted to lead, the ways in which this was possible or not, and what they did or felt unable to do as a consequence of this. Moreover, we added that we wanted to explore if and how there were new and emergent forms of racism. In itself this was a challenge but it was essential to building frank relationships with participants. We followed this by talking about their experiences of migration and their current circumstances before thinking together about 
what to analyse further and how. This included discussion of what participants and/or the researchers would assemble or record for future meetings. Participants undertook the work, or adapted the agreed plan, in order to explore the topic of our research. We then met them on several occasions to discuss what had been collected, swapping ideas, the analysis and synthesis of which informed further material collection by participants if they wished. Inherent in this process was the partial collapse of the fieldwork/analysis dichotomy that separates fieldwork with participants from the analytical critical reckoning done solely by researchers. This argument can be illustrated through a detailed account of how such a relationship unfolded with a participant called Dorothy.

\section{Dorothy goes to Buckingham Palace}

Unlike our first meetings with many other participants, we met Dorothy in an official-looking interview room of a Third Sector organisation through which Author A had made contact with her. It was an archetypal 'quiet place' like the one mentioned at the beginning of this article, perfect for recording but not very sociable. From the opening exchanges it became clear that Dorothy was used to being asked questions to extract information from her. The research process at this point resembled the 'interviewer as information miner' stance that we had wanted to distance ourselves from: 
Author A: Oh right, okay, and why was it they [the Police] helped you to find [name of welfare organisation], what was the reason?

Dorothy: To help me claim benefits for him [points to her baby], yeah, and my immigration status.

Author A: Okay, and how long have you been in Britain?

Dorothy: Almost three years now.

Author A: Three years almost, okay. And I think you told me before but what was it, you came from Ghana was it?

Dorothy: Yeah, Ghana.

Author A had wanted to talk about less personal issues first given he had not met her before. However, enquiries of a 'general' nature about circumstances and how Dorothy made contact with the welfare organisation quickly led to intensely personal talk. After less than two minutes we started discussing her separation from her mother and the death of a beloved grandma:

Dorothy: My mum kind of got problems with me being pregnant because I was 16 at that time. 
Author A: Oh yeah.

Dorothy: So she left and after that I don't know where she is, I'm kind of stuck in the country trying to find my way out to support myself and my little boy.

Author A: Okay, so you came ...

Dorothy: On a visit.

Author A: On a visit, so like a family visit or something was it?

Dorothy: Yeah, family visit.

Author A: To come to the UK, and while you were here you gave birth and then you weren't able to stay in contact with your mum.

Dorothy: Yeah, because she left before I have him.

Author A: And that meant that you were sort of-

Dorothy: I got stuck here. 
Author A: Got stranded.

Dorothy: Yeah, because back home I haven't got anyone. My grandma was living with my mum, she died. Yeah, so I was like with my mum and my family down here in London.

Author A: There was no family back there?

Dorothy: Yeah.

Author A: And how old were you then?

Dorothy: I was 16 at that time when I first arrived in the country.

During the course of our initial meeting Dorothy explained more about her situation, for example how immigration surveillance had limited her opportunities in different ways and the consequential effects, such as material shortages, that she and her son had experienced. The study was critical of the immigration framework, and this was inherent in the questions Author A asked. This, combined with a genuine attempt to understand her perspective rather than merely collating information on factual movements and circumstances, might explain why Dorothy was 
keen to continue participating in the study. However, what appeared to be replayed in this first meeting was a sense of the interviewer as miner, as exemplified by the narrative extraction model.

Author A and Dorothy talked over whether and how she might want to observe certain parts of her life and why it might be useful for the study. Dorothy was keen to use a camera, which Author A was able to supply, and later on she also assembled and spoke about personal objects she owned, documents she collected and writing she did. Initially, Author A suggested that Dorothy should decide what aspects of her daily life she wanted to capture for our study, which might relate to the aims of our research. Dorothy was unsure what to examine and asked for suggestions; he suggested exploring through photographs the way in which her immigration status affected where she went and what she was able to buy. At this point we had moved away from data extraction. The process of suggesting a task was similar to elicitation methods, albeit here as an example rather than a condition of participation. Nonetheless, it was also different from elicitation in that we had been open to what Dorothy might want to explore within the parameters of the overall research.

Almost exactly two months later we met again, this time, at her invitation, at her house in Croydon. Instead of taking photos of her daily 
life as Author A suggested she had gone sightseeing with the camera.

[Dorothy's photograph of Buckingham Palace]

Dorothy explained why she took this photo: 'Yeah, because when I was in Ghana my grandma did come here - I think I wasn't born at that time and she told me a lot about Buckingham Palace [and] how brilliant it is'. This appears to have little connection with the concerns of our study. However, Dorothy had made a specific choice that expanded our attention.

Previously Dorothy had been arrested while attempting to obtain a job using a friend's passport and national insurance number. She had no access to money and was prevented from working because her original tourist visa did not allow it. Her tourist visa had also expired and the clandestine circumstances of her life meant she was scared to move in particular places like Buckingham Palace and elsewhere because there were lots of police there: 'Because before I got arrested I was fear, it was even a fear when I see a police person pass and I'm really feared to go pass through that place. It's the fear of that, like the fear of being close to the police or something'. She added: 'In that area [speaking of London tourist sites], because what I find is like people, I think especially the blacks, sometimes, like the immigrants amongst them, like the few, it's like oh what am I going to do there, what am I going to do? It's like they 
sometimes fear because they don't know what is going to happen to them when they go to certain places'.

However, Dorothy had been near Buckingham Palace before: 'because like the first time that I passed through there was the day that I was coming back from court after I was arrested. When I was arrested the following day I went to court. So I was a bit sad'. Dorothy went on to relate how her situation had improved as a migrant support agency had connected her with a solicitor who had helped her obtain a Temporary Leave to Remain (TLR) visa. Her son was enrolled at nursery and she had started a predegree HND nursing course, for which her uncle was paying the fees, as she wanted to become a midwife after her grandma imbued her with how wonderful midwifery was. Dorothy explained how the TLR had improved her conditions. She now had an official immigration status meaning that, although she and her son could be detained at any time should the authorities decide, she was not subject to incarceration following a random police check on her official status in the country, as was the case when she had no valid visa at all. During this study she went to Buckingham Palace, the place her grandma had inspired her to visit, which she previously felt unable to do because of a fear of immigration surveillance: 'So when I saw it it's like oh that's the past. Later on, me being in a happy mood and then going there to take pictures of them made me much happy.' 
In our conversation on that day Dorothy spoke of how, prior to this, she was in a situation where she saw no future for herself and her son, and felt constrained in her present life, because of a lack of educational opportunities for her and her son combined with a lack of money. These constraints literally limited her spatial movements, meaning she spent lots of time at home thinking of the past and her grandma. She still does think of her grandma, but because her present circumstances have changed although they remain precarious, this is from a different perspective. Dorothy is more active now and can envisage a good future, so that when she revisits the memory of her grandma this now inspires her in her wish to become a midwife and to remember the life-lessons her grandma taught her.

Dorothy's snapshot of Buckingham Palace is a transformative gesture shifting the analytical terms from a life circumscribed by the immigration system toward a different story about herself, her past and her family. She trusted us with a glimpse into a freer life and the photograph is perhaps a small act in the direction of a happier future, a signal of a fuller expression of the kind of person Dorothy is and against a sense of loss and abandonment. Dorothy's story of being enchanted at Buckingham Palace contains so much more than is captured in the abstract notion of 'the immigrant.'

We wanted to foster a form of research dialogue that enabled our 
participants to shift the analytical terms of reference and by choosing to take photos of Buckingham Palace rather than following Researcher A's suggestion of what photos to take she did. Our research contributes to the development of participatory research where non-specialist researchers conduct research and where "there is shared ownership of research projects, community-based analysis of social problems and an orientation toward community action" (Kemmis and Taggart 2008:273). In our study, we grappled with a means to develop a way of listening and talking that recognised that there were limits to the insight of both researchers and participants, and tried to play them off against one another to see what each insight could bring when brought together in dialogue.

This involved analysing material together with participants and being attentive to their interpretations. At the same time we were methodologically rigorous about the reliability of the accounts that were offered. Sanders and Cuneo (2010) emphasise the need for research teams to code and crosscheck their coding frequently. Our dialogue necessitated a continual process of listening, crosschecking and sharing meaning, not only between researchers but also when meeting with participants. This was grounded by an attempt to re-shape the dynamic of dialogue between researcher and participant, rather than making a technical adjustment to transcript-reading following an interview. Inglis (2010) takes an approach which eschews the technical analytical framework of grounded analysis, instead favouring observation that looks for 'clues' informed by a prior 
understanding of theories and concepts. We too eschewed technical analytical frameworks but did our searching for clues in direct dialogue with participants. The technical work of sifting, categorizing and making sense was carried out through dialogue entailing the cross referencing of previous conversations and material. In Dorothy's example it expanded our insight concerning how immigration laws and societal structures limited the spaces where some could work and move. This 'shrunk' the possibilities of life against which Dorothy was asserting herself. We found this particularly important given how theoretical frameworks exploring contemporary points of immigration surveillance are often influenced by network theory in mapping the mobility of the border omitting the function this has in asphyxiating life (see for example Mezzadra and Nielson 2008).

To gain this insight we adopted and argue here for a position different from the idea of speaking for migrants, opting instead for a sociable process of travelling alongside in dialogue. Sara Ahmed has commented that the notion of "speaking for the other [...] is premised in fantasies of absolute proximity and absolute distance" (Ahmed 2000: 166). This kind of ventriloquism both silences and confines that subject for whom the author speaks. We are arguing that research needs to be a more sociable process in which voice itself becomes a value. Nick Couldry (2010:2) writes: "Valuing voice then involves particular attention to the conditions under which voice as a process is effective, and how broader forms of 
organisation may subtly undermine or devalue voice as a process".

Voice as a process is devalued when the interviewer is viewed as a 'data miner' or when writing is the exclusive province of a distant analyst who has scrutinized a life s/he inhabits only momentarily. What we learned from Dorothy is not some inner secret about whether she is 'legal' or 'illegal' or 'deserving' or 'bogus'. Rather, travelling with Dorothy on her trip to Buckingham Palace, we are afforded a glimpse into her free life and not just the aggrieved one that brought her to our initial attention. As a result this offered us an opportunity to understand the intersecting horizons of her past and present circumstances. Dorothy's gift to us as researchers is that we come to appreciate an expanded sense of what it means to be human in the $21^{\text {st }}$ Century.

\section{Not On but With}

Our argument for making methods sociable emphasises dialogue and reconfiguration of the relationship between participant and analyst. Andrea Cornwall and Rachel Jewkes (1995:1674) commented: "Slowly and often painfully conventional researchers are coming to realise that working with the poor and voiceless is infinitely more rewarding than working on them". They also argue that the move to see researching 'not on but with' participants involves institutional changes within the wider culture of 
research and writing. We conclude by discussing how the sociable turn raises issues about research ethics and the nature of authorship that are part of this culture. To do this we return to where we started, with our meeting with Charlynne in The Eatery to discuss a draft journal article.

The article, entitled 'New Hierarchies of Belonging', was about her life and included her creative writing and a poem about her encounter with a black immigration officer. The nature of our dialogue with Charlynne had blurred the boundary between 'research data' and 'analytical writing'; our analysis involved talking with her about material which the research had produced whilst also in itself being a recorded part of that material to be used for further analysis between us all.

Charlynne had not co-written the paper but had contributed in an important way to its authorship. Carrying through our commitment to work with migrants rather than on them we proposed that the authorship attribution for the article should include her name. We agreed that the author's attribution should read "Author B and Author A with Charlynne Bryan". The article was submitted to an academic journal and it was accepted. The story of what unfolded next reveals the kinds of institutional limitations hinted at by Cornwall and Jewkes.

During the production process the status of Charlynne as an author was queried and it was suggested a pseudonym could be provided for her, as 
this was 'standard practice when discussing research participants in print'. Additionally, the publisher queried the 'ethical and permissions issues' involved in revealing a participant's identity. We said we were making an ethical point by giving Charlynne credit and that she herself did not want a pseudonym to be used. This incident revealed a presumption - widely held in the culture of research - that good ethical practice requires automatic anonymity for participants (see also BSA 2002 Section 34). This un-thinking default position, we argue, is an anxious symptom of ethical hypochondria, which limits the opportunities to rethink authorship and innovate new formats for research, particularly in the context of digital hyper-connection. This inhibition is curious given that, within social networking sites like Facebook and YouTube, people are 'broadcasting themselves' to an unprecedented degree.

In the end the publisher relented and the article was published with Charlynne's attribution (self-reference removed). There was one final problem. The publisher wanted to know at which educational institution Charlynne was based. In order to be credited it is presumed you have to have a university association. This again alluded to tacit divisions assumed within the culture of research concerning separations between both data and analysis and also participants and analysts that we were trying to re-configure.

In addition, the fact that Charlynne used her own name enabled other 
kinds of circulations to occur. Prior to publication we emailed a draft of the article to Avery Gordon, a sociological colleague, who was looking for a contemporary account of migration for her teaching at University of California at Santa Barbera (UCSB). Our draft paper was read by 80 UCSB students. Our account caught the imagination of one of the students - Daniella Florant - who like Charlynne had family in Dominica. Daniella Googled Charlynne's name to find out more background about her in preparation for her seminar presentation. She found some YouTube footage of Charlynne reading her poetry on the University of East London website, which she included in her PowerPoint. Imposing 'automatic anonymity' would have foreclosed the possibility of such circulations of imagination and connection, and limited the social life of this 'data'. Daniella and her classmates introduced Charlynne respectfully with a short academic biography as one of the three authors of the article. Charlynne was presented as a serious writer and poet; as Avery commented in an email: "the journal's question about her 'institutional affiliation' would have seemed both strange and disrespectful" (personal communication $25^{\text {th }}$ August, 2012).

As Paul Rabinow (2003:115) has written the "main mode of regulation now is "ethical". The broader issue is that we do not as yet have an ethical language supple enough to facilitate the possibilities exemplified above. Narrow ethical concern is closing down sociological practice rather than 
opening it out to new possibilities to challenge the often-damaging ways in which 'the migrant' is figured. What Charlynne's example points towards is the possibility for sociology to travel in a hyper-connected world, to connect and re-enchant and, from the perspective of the UCSB student, to see the likeness of herself in another. Daniella Florant commented via email: "Being able to see Charlynne's face, hear her speak, and know her story added a personal connection [...] and made it much more real than just words on paper" (personal communication, $27^{\text {th }}$ August, 2012).

\section{Conclusion: Reconfiguring Qualitative Research}

Our argument for sociable methods requires a reconfiguring of the dominant paradigm of qualitative research and processes of both data extraction and elicitation that are situated within it. This de-stabilises the clear division of labour between researchers and participants, which also challenges the position of the sociologist. This kind of openness might seem frightening or worrying for researchers because it challenges our hold on producing knowledge. We argue that it is a risk worth taking. Our experience of experimenting with dialogic research enabled insights to come into view that had been hidden within our blind field. Also, our participants do not want us to surrender our expertise. We found quite the reverse. What they wanted was to bring our erudition and overview into dialogue with their own hunches and insights, in a spirit of trust and 
mutual respect.

What we did in this project was to allow the young people we worked with to be included in the problem solving process as authors. Important questions remained and had to be negotiated about the point at which an output becomes something which is co-authored by researchers and participants, how this re-defines the researcher/participant relationship, and which forms of authorship/contribution evolve that recognise the particularity of a researcher's authorial voice with the dialogic knowledgemaking role of participants (see also Photovoice 2008).

Savage and Burrows (2007) have precipitated an important debate about sociology's 'empirical crisis'. What kind of jurisdiction can academic social research have in a society already producing and analysing information on an unprecedented scale? One way out of this is to develop sociable research methods that are distinctive because they are committed both to achieving transformative ways of producing knowledge and creating a kind of public around a problem composed of researchers, participants and audiences. Following Andrew Abbott (2007: 96) we are also arguing that sociable methods should have an aspiration to "cherish and develop the lyrical voice" that is compelling and accessible.

Anxiety within universities regarding the moral dimension of academic research, we argue, is producing 'ethical hypochondria' and unthinking 
conservatism. Imposing 'compulsory anonymity' for research participants can be limiting because it makes qualitative researchers less able to develop new forms of collaborative authorship and research craft. It also means that researchers are less able to embrace the opportunities that digital culture affords to make data and insight travel and connect with interested global audiences.

There are many cases where the forms of facilitative dialogue we have argued for may not be appropriate e.g. working with racists or Holocaust deniers or powerful corporate executives. However, for marginalised groups, sociable methods offer the potential to produce deep insight while bringing them into a conversation with sociology as both participants and authors. A response made all the more pertinent given the limits sociological theory encounters when trying to analyse new formations of inequality and the experience of migration which challenges its limits.

Charlynne's surprise on reading our paper captures precisely the qualities we want to argue for. When she asked 'How do you know that?' there was a sense of wonder and re-enchantment in her question, as if reading the paper helped her see her experience of life anew. Similarly, for us as researchers, when Dorothy said why she had photographed Buckingham Palace it caught our imagination. Listening to her helped us rethink existing sociological ideas and theories but also made us see her life beyond the confines of her status as an immigrant. Research here is about 
researchers and participants putting up-close experiences at a distance so that they can be reincorporated anew. The result is a mutual transformation, whether it be Charlynne's understanding of herself or our appreciation of the limits of sociological theory. A reanimation of life is produced precisely through such an exchange of social horizons and imagination. This kind of sociable approach is dedicated to seeing the confinements contained in society's blind field, or hearing the injustice buried by noise or hidden in silence and bringing it into public conversation. 